\title{
A Large Sky Survey with MeerKAT
}

\author{
Mário G. Santos, ${ }^{* 1,2}$ Philip Bull, ${ }^{3,4}$ Stefano Camera, ${ }^{5}$ Song Chen, ${ }^{1}$ José Fonseca, ${ }^{1}$ \\ Ian Heywood, ${ }^{6}$ Matt Hilton, ${ }^{7}$ Matt Jarvis, ${ }^{1,6}$ Gyula I. G. Józsaa ${ }^{2,8,9}$, Kenda Knowles, ${ }^{7}$ \\ Lerothodi Leeuw, ${ }^{10}$ Roy Maartens, ${ }^{1,11}$ Eliab Malefahlo, ${ }^{1}$ Kim McAlpine, ${ }^{1}$ Kavilan \\ Moodley, ${ }^{7}$ Prina Patel, ${ }^{1,2}$ Alkistis Pourtsidou, ${ }^{11}$ Matthew Prescott, ${ }^{1}$ Kristine \\ Spekkens, ${ }^{12}$ Russ Taylor, ${ }^{1,13}$ Amadeus Witzemann ${ }^{1}$ and Imogen Whittam ${ }^{1}$ \\ ${ }^{1}$ Department of Physics \& Astronomy, University of Western Cape, Cape Town 7535, South Africa \\ ${ }^{2}$ SKA SA, The Park, Park Road, Cape Town 7405, South Africa \\ ${ }^{3}$ California Institute of Technology, Pasadena, CA 91125, USA \\ ${ }^{4}$ Jet Propulsion Laboratory, California Institute of Technology, Pasadena, California, USA \\ ${ }^{5}$ Jodrell Bank Centre for Astrophysics, The University of Manchester, Manchester M13 9PL, UK \\ ${ }^{6}$ Department of Physics, Oxford University, Oxford OX1 3PU, UK \\ ${ }^{7}$ Astrophysics \& Cosmology Research Unit, Univ. of KwaZulu-Natal, Durban 4041, South Africa \\ ${ }^{8}$ RARG, RATT, Rhodes University, Grahamstown, 6140, South Africa \\ ${ }^{9}$ Argelander-Institut für Astronomie, Auf dem Hügel 71, D-53121 Bonn, Germany \\ ${ }^{10}$ School of Interdisciplinary Research \& Graduate Studies, UNISA, Pretoria 0003, South Africa \\ ${ }^{11}$ Institute of Cosmology \& Gravitation, University of Portsmouth, Portsmouth PO1 3FX, UK \\ ${ }^{12}$ Department of Physics, Royal Military College of Canada, Kingston, ON K7K 7B4, Canada \\ ${ }^{13}$ Department of Astronomy, University of Cape Town, Cape Town 7701, South Africa \\ E-mail: mgrsantos@uwc.ac.za
}

We discuss the ground-breaking science that will be possible with a wide area survey, using the MeerKAT telescope, known as MeerKLASS (MeerKAT Large Area Synoptic Survey). The current specifications of MeerKAT make it a great fit for cosmological applications, which require large volumes. In particular, a large survey over $\sim 4,000 \mathrm{deg}^{2}$ for $\sim 4,000$ hours will potentially provide the first ever measurements of the baryon acoustic oscillations using the $21 \mathrm{~cm}$ intensity mapping technique, with enough accuracy to impose constraints on the nature of dark energy. The combination with multi-wavelength data will give unique additional information, such as the first constraints on primordial non-Gaussianity using the multi-tracer technique, as well as a better handle on foregrounds and systematics. The survey will also produce a large continuum galaxy sample down to a depth of $5 \mu \mathrm{Jy}$ in L-band, unmatched by any other concurrent telescope, which will allow to study the large-scale structure of the Universe out to high redshifts. Finally, the same survey will supply unique information for a range of other science applications, including a large statistical investigation of galaxy clusters, and the discovery of rare high-redshift AGN that can be used to probe the epoch of reionization as well as produce a rotation measure map across a huge swathe of the sky. The MeerKLASS survey will be a crucial step on the road to using SKA1-MID for cosmological applications, as described in the top priority SKA key science projects.

MeerKAT Science: On the Pathway to the SKA,

25-27 May, 2016,

Stellenbosch, South Africa

* Speaker. 


\section{Rationale}

A new generation of radio telescopes with unprecedented capabilities for astronomy and tests of fundamental physics will enter operation over the next few years. Thanks to their large survey speeds, they present a unique opportunity to probe the Universe using large sky surveys. These will deliver measurements of the large-scale structure of the Universe across cosmic time, a crucial ingredient in pushing the current limits of precision cosmology and exploring new cosmological models, thanks to their huge 3-dimensional information content.

The key example is SKA1-MID, the first phase of the SKA, to be set in South Africa, which will deliver competitive and transformational cosmology through a series of measurements using large sky surveys $[1,2,3,4]$. However, in the immediate future, MeerKAT ${ }^{1}$, the SKA precursor in South Africa, will have the capability to produce high impact cosmological constraints from a large sky survey. Several factors contribute to this: i) the final MeerKAT specifications deliver excellent survey speed (low noise, large primary beam), exceeding initial expectations; ii) shorter baselines make MeerKAT a better fit for large, shallow surveys, instead of deep surveys that may suffer from confusion noise; iii) MeerKAT's dish design, with single pixel feeds, provides a stable, well-tested set-up that should allow for a better handle on calibration issues, an important consideration for extracting the small cosmological signal (in particular using the auto-correlations). A large amount of the science that we plan to do will benefit greatly from a multi-wavelength approach. Therefore, "full sky" surveys are not a requirement, as we can achieve most of the science with smaller areas that are covered simultaneously by surveys at other wavelengths. Finally, the proposed survey will pave the way for using the full SKA1-MID array for cosmology - one of the SKA's key science projects.

In recognition of MeerKAT's potential as a cosmological survey instrument, we propose a survey of $\sim 4,000 \mathrm{deg}^{2}$ in $\sim 4,000 \mathrm{hr}$ over the same sky area as the Dark Energy Survey, DES (www.darkenergysurvey.org), which will provide a wealth of multi-wavelength data (note however that the final survey footprint is still being decided). This survey, called MeerKLASS (MeerKAT Large Area Synoptic Survey), will allow for a variety of scientific breakthroughs in a completely new regime:

1. Possibly the first ever detection of baryon acoustic oscillations (BAO) and redshift space distortions (RSD) through the $21 \mathrm{~cm}$ intensity mapping technique, which will allow novel cosmological constraints to be placed on dark energy and alternative theories of gravity. This will be achieved in both auto-correlation and cross-correlation with other surveys, the latter guaranteeing a much cleaner signal with regard to systematics. Besides cosmology, these measurements will also constrain the HI mass function and its evolution, as well as the clustering bias of HI galaxies.

2. The first ever constraints on primordial non-Gaussianity using the multi-tracer technique, improving on current limits.

3. Detect $\sim 10^{7}$ (mostly star-forming) galaxies in continuum, that can be used for several statistical tests in cosmology.

4. Probe the AGN/ SF galaxy populations up to large masses, and even find the rarest high-redshift AGN, which can be used to probe the EoR through $21 \mathrm{~cm}$ absorption.

5. Provide a large sample of galaxy clusters, and a study of radio halos and relics in clusters.

\footnotetext{
${ }^{1}$ https://www.ska.ac.za/science-engineering/meerkat
} 
6. Detect thousands of sources which can be used to investigate associated HI absorption systems.

7. Produce a rotation measure map across a large patch of the sky.

8. Perform transient searches.

MeerKLASS will use MeerKAT's L-band receivers (900-1670 MHz), in order to achieve a point source sensitivity in continuum of $\sim 5.3 \mu \mathrm{Jy}$. This is assuming about $25 \%$ band loss to RFI, and taking into account the expected confusion (which should be low at this level). The specifications used in our calculations can be found at: http://meerkat2016.ska.ac.za/Files, where we used the full 64 dishes. In the following analysis, we will sometimes consider other options (such as UHF-band or different survey areas/time) in order to justify our choices.

We aim to use the single-dish auto-correlations and interferometric data from MeerKAT simultaneously. The single-dish survey will require a particular calibration approach. First, we propose a fast scanning strategy, in order to suppress the effect of gain variations on the required angular scales for cosmology. Second, we plan to use the MeerKAT noise diodes to inject a frequent, stable signal that can be used for calibration. The interferometer data will be taken at the same time, using an on-the-fly observing mode. This again will use a novel calibration technique, where the full dish primary beam will be included. Note also that cross-correlating the intensity between dishes can also help with systematics and the noise bias. In the next sections, we describe in detail the science that can be done, and the reasoning for the requested sky area and sensitivity.

\section{Cosmology}

MeerKLASS aims to provide some of the first constraints on cosmology using the new generation of radio telescopes. With the same data, we will effectively run two different cosmological surveys: $\mathrm{HI}$ intensity mapping and a continuum radio galaxy survey. Combined with multi-wavelength information, these will allow exquisite constraints on several key cosmological parameters.

\subsection{HI intensity mapping}

Typically, large-scale structure probes in cosmology use galaxy surveys with spectroscopic or photometric redshifts in the optical or near-infrared. These are threshold surveys in that they set a minimum flux above which galaxies can be individually detected. For the radio, we will have to rely on the $\mathrm{HI} 21 \mathrm{~cm}$ signal to obtain such redshifts. But this emission line is quite weak. The next generation of radio telescopes will only achieve the required sensitivities to detect HI galaxies over small volumes not useful for cosmology. In fact, we will have to wait until SKA2 to achieve the sensitivity to do game-changing cosmology with an HI galaxy survey [5].

The intensity mapping technique relies on observations of the sky intensity over a large number of pixels instead of trying to detect galaxies. For a reasonably large 3D pixel (in solid angle and frequency interval), we expect to have several HI galaxies in each pixel so that their combined emission will provide a larger signal. Moreover we can use statistical techniques, similar to those that have been applied to CMB experiments, to measure quantities in the low signal to noise regime. By not requiring the detection of individual galaxies, the sensitivity requirements are much less demanding. Intensity mapping transfers the problem to one of foreground cleaning: how to develop cleaning methods to remove everything that is not the HI signal at a given frequency, which also impacts on the calibration requirements of the instrument [2]. 
The GBT produced the first (tentative) detection of the cosmological HI intensity signal by cross-correlating with the WiggleZ redshift survey [6, 7, 8]. Many other intensity mapping experiments are underway or planned for the near future, such as BINGO, CHIME, TIANLAI and HIRAX.

\subsubsection{Detecting the HI intensity mapping signal with MeerKAT}

The first aim of the intensity mapping survey will be to measure the HI power spectrum, something that has not been achieved yet. In addition, we will take advantage of multi-wavelength coverage (e.g., BOSS, DES) to detect the signal in cross-correlation. This will reduce systematics and potentially make the detection even easier. The MeerKLASS survey can be performed in the L-band $(0<z<0.58)$ or UHF-band $(0.4<z<1.45)$. The noise properties of an intensity mapping survey like MeerKLASS have been described in detail in [2,9].

The HI signal and measurement errors expected from MeerKLASS (4000 deg ${ }^{2} \mathrm{sky}$ area, $4000 \mathrm{hr}$ observation time) are shown in Fig. 1, top left, for a redshift bin of width $\Delta z=0.1$ centered at $z=0.5$. Here RSD have been neglected. Assuming a $\Lambda$ CDM expansion history, the only unknown in the power spectrum is the $\mathrm{HI}$ density times the bias, $\Omega_{\mathrm{HI}}(z) b_{\mathrm{HI}}(z)$. Employing a Fisher matrix analysis, [10] has shown that the constraints on this quantity will be more than one order of magnitude better than current constraints from galaxy surveys, intensity mapping, and damped Lyman- $\alpha$ observations [11].

Detection can also be achieved by cross-correlating with optical surveys [8]. The advantage is that systematic effects and foregrounds that are relevant for one survey but not for the other are expected to drop out in the cross-correlation. Figure 1, top right, shows the expected signal and errors for a BOSS DR12-like spectroscopic sample [12] at $0.2<z<0.4$. The assumed sky overlap is $500 \mathrm{deg}^{2}$ with $500 \mathrm{hr}$ MeerKAT time. The forecast constraint is $\delta\left(\Omega_{\mathrm{HI}} b_{\mathrm{HI}} r\right) /\left(\Omega_{\mathrm{HI}} b_{\mathrm{HI}} r\right)=0.02$ [10]. Figure 1, bottom left, shows the expected (angle-averaged) signal and errors for a DES-like photometric sample [13], with $4000 \mathrm{deg}^{2}$ sky overlap and $4000 \mathrm{hr}$ MeerKAT time. Finally, Fig. 1, bottom right, shows the expected cross-correlation signal and errors between the HI density field at $z_{f}=0.3$ and the lensing convergence $\kappa$ of background sources at $z_{b}=1$, using MeerKAT and DES (for details, see [9]).

\subsubsection{Baryon acoustic oscillations and redshift space distortions}

BAO currently provide the most powerful probes of the Universe's expansion history $\left(D_{A}, H\right)$, while RSD do the same for the rate of growth of structure $\left(f \sigma_{8}\right)$. MeerKLASS aims to make the first detections of $\mathrm{BAO}$ and RSD with enough signal to noise to constrain the cosmological model. It will in fact provide better constraints than any current galaxy survey.

Figure 2 shows the expected signal to noise from BAO and RSD for different parameters, as a function of sky area and for a few observation times, at $z=0.4$ and $z=1.0$ (with a $\mathrm{z}$ bin of 0.1). The forecasting procedure follows the approach described in [14] (see also [10]). Looking in particular at the constraints on $A$, the overall BAO amplitude (left), we see that the signal to noise is better in the L-band. Moreover, 4,000 hr and 4,000 $\mathrm{deg}^{2}$ is seen as a good balance. For the same $4,000 \mathrm{hr}$, more sky area will be better, but not so much better as to offset the penalty on the other science, such as the continuum science case. On the other hand, 2,000 hr provides a S/N below 2 which makes it a risky option in terms of making a clean signal detection. Note also that we want 

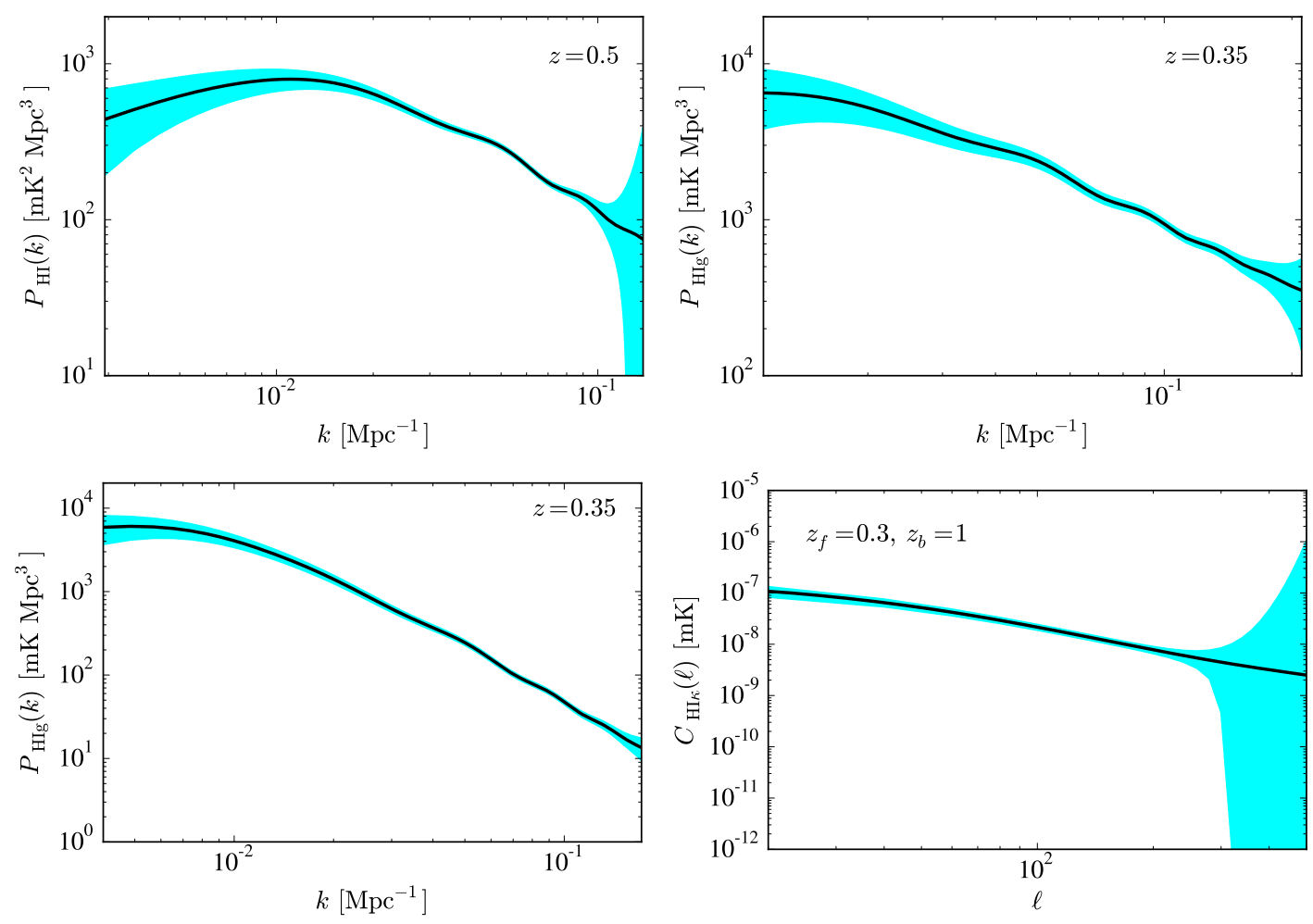

Figure 1: HI detection with MeerKLASS, showing the expected signal power spectrum (black solid) and measurement errors (cyan). Top left: HI auto-correlation. Top right: Cross-correlation with BOSS DR12-like sample. Bottom left: Angle-averaged cross-correlation with DES-like survey. Bottom right: DES galaxies (at $z_{b}=1$ ) as background sources to detect the cross-correlation between the HI density field (at $z_{f}=0.3$ ) and lensing.

to use sky areas with good multi-wavelength coverage, which will essentially set the maximum available area to about $6,000 \mathrm{deg}^{2}$ for the next six years. The upper right panel in Fig. 2 shows that detection of the growth rate with MeerKLASS will be possible with a signal to noise of almost 40 in the L-band.

\subsubsection{Multi-tracer constraints on primordial non-Gaussianity}

Measuring primordial non-Gaussianity (PNG) provides a powerful probe of inflation models. The local type of PNG is parametrized by an amplitude $f_{\mathrm{NL}}$ and current state of the art constraints on PNG are delivered by the Planck experiment, $\sigma\left(f_{\mathrm{NL}}\right)=6.5$, using measurements of the bispectrum. PNG also leaves a 'frozen' imprint in the power spectrum on very large scales by introducing a scale-dependent clustering bias $[15,16]$. For a single tracer of the dark matter distribution, this signal is eroded by cosmic variance, and even the next-generation ultra-large survey volumes are unable to achieve $\sigma\left(f_{\mathrm{NL}}\right)<1$ [17].

Cosmic variance can be beaten down using multiple tracers, and this is applied to MeerKAT and DES in [18]. It is shown that the multi-tracer technique can achieve errors on $f_{\mathrm{NL}}$ better than those of Planck, even with a conservative assumption on the overlap sky area between MeerKAT and DES $\left(3,000\right.$ or $\left.4000 \mathrm{deg}^{2}\right)$, and with a maximum of $4000 \mathrm{hr}$ integration time $\left(\sigma\left(f_{\mathrm{NL}}\right) \sim 3.6\right.$ 

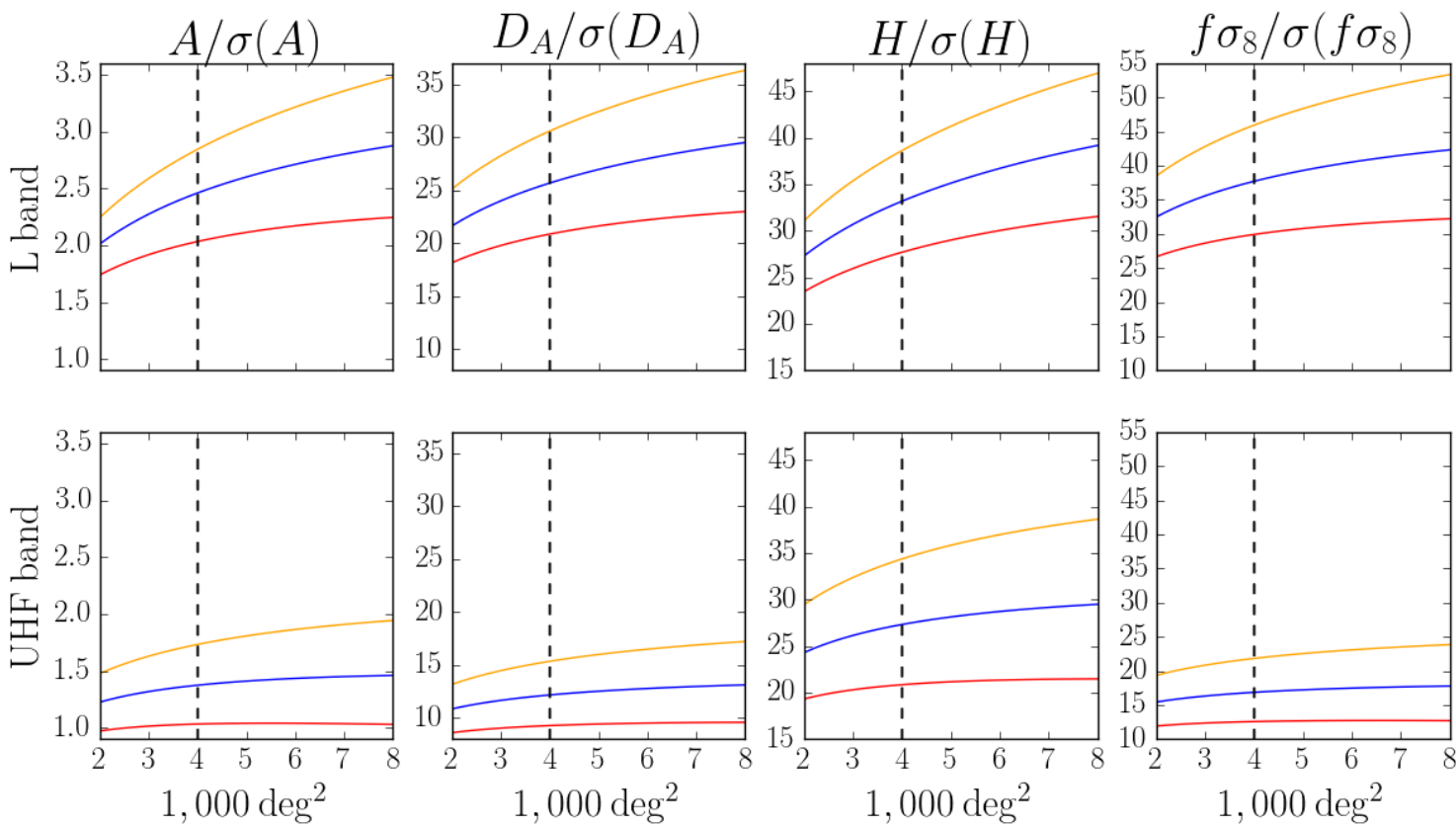

Figure 2: Signal to noise as a function of survey area. Columns from left to right show: BAO amplitude parameter $A$, angular diameter distance $D_{A}$, Hubble rate $H$, growth rate $f \sigma_{8}$. Curves are for: 2,000 (red), 4,000 (blue), 8,000 hr (orange). Upper row: $z=0.4$ in L-band, lower row: $z=1.0$ in UHF-band (redshift bin size $=0.1$ ). Vertical dashed lines indicate $4,000 \mathrm{deg}^{2}$.

in L-band and 2.3 in UHF). MeerKLASS can therefore deliver the best constraints on $f_{\mathrm{NL}}$ before SKA, Euclid and LSST deliver data.

\subsection{Cosmology with a continuum galaxy survey}
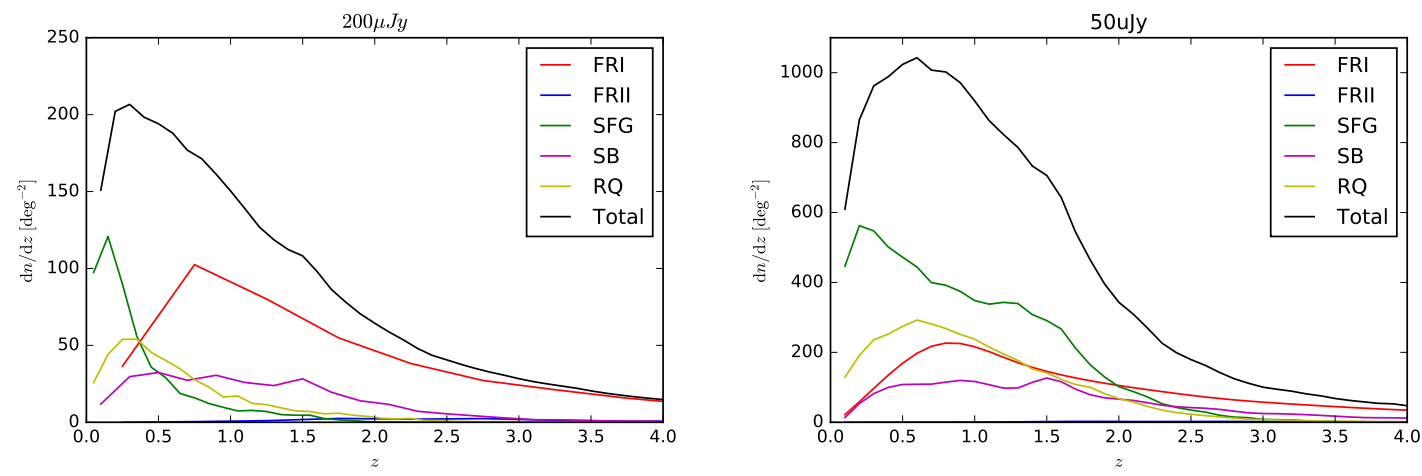

Figure 3: Redshift distribution of continuum sources detected at 200 (left) or $50 \mu \mathrm{Jy}$ ( right, the MeerKLASS flux cut) for various radio galaxy populations (different colours) and the total (black). Most MeerKLASS galaxies will be star forming.

A continuum survey can be done simultaneously with an $\mathrm{HI}$ intensity mapping survey. Contrary to line surveys, requiring high resolution in frequency, these surveys integrate over a con- 
tinuous interval in frequency $(\sim 300 \mathrm{MHz})$, providing high sensitivity and the detection of a large number of radio galaxies $\left(>10^{8}\right.$ with SKA1). Unlike wide-field optical surveys, radio continuum emission is unaffected by dust, and AGN and galaxies will be detected to high redshifts. MeerKLASS will be able to detect almost 10 million radio galaxies (a factor of 10 more than current surveys), which will allow us to impose stringent constraints on dark energy.

Since continuum radio surveys reach higher redshifts $(z \gtrsim 2.5)$ than optical/near-IR counterparts, they are in principle ideal to track the evolution of dark energy. Figure 3 shows the redshift distribution per square degree of various radio galaxy populations (coloured curves) that will be detected by a continuum survey with a flux cut at 200 (left) or $50 \mu \mathrm{Jy}$ (right). The latter is the $\sim 10 \sigma$ level for MeerKLASS, while the former corresponds roughly to the planned ASKAP EMU survey $\sim 10 \sigma$ flux cut.

The distributions have a high- $z$ tail, with non-negligible source number densities as deep as $z \sim 4$. However, this major advantage of radio continuum for cosmology is spoiled by the featureless synchrotron spectrum, which does not allow for the measurement of redshift. To overcome this, we can use redshift information from cross-identifying sources via observations at different wavelengths [19]. Using 5 photometric bins in $0.01<z<0.8$ and then a large bin containing all the remaining high- $z$ galaxies, we forecasted the precision of a continuum angular power spectrum in constraining the dark energy equation-of-state parameters $w_{0}$ and $w_{a}$, representing the present-day value and the evolution with redshift. For MeerKLASS, the standard dark energy figure of merit can reach a value of $\sim 1000$.

\section{Galaxy evolution}

Radio continuum emission at $\sim 1.4 \mathrm{GHz}$ is dominated by synchrotron emission from AGN and SF galaxies, and provides a clear view of the emission mechanisms, free of dust obscuration.

\subsection{AGN}

The depth and area of MeerKLASS is ideally suited for investigating evolution of AGN and the impact they have on their host galaxy and wider environment. The most powerful AGN $\left(L_{1.4 \mathrm{GHz}}>\right.$ $10^{25} \mathrm{WHz}^{-1}$ ) evolve strongly over cosmic time, with number density increasing by a factor of $\sim 1,000$ from $z=0$ to 2 , and then declining beyond $z \sim 3$. For lower luminosity AGN, the picture is more uncertain, with some studies finding little or no evolution [e.g. 20, 21] and others finding more significant evolution [e.g. 22]. Lower luminosity AGN dominate the AGN feedback required by the latest simulations of galaxy evolution, and understanding their evolution as a function of accretion rate and environment is crucial for our general understanding of massive galaxy evolution.

MeerKLASS will provide the data necessary to answer this question, particularly when combined with the wealth of multi-wavelength data available over the proposed fields. Using the $S^{3}$ simulation as a guide, we will discover $\sim 50,000$ FRI-type galaxies at $z<1$ and measure the evolution out to $z \sim 2$, where it has yet to be measured with any precision. Furthermore, using optical and near-IR data (DES, KIDS, VIKING, VHS), we will relate the radio emission to the host galaxy properties and the accretion rate of the AGN, using mid-infrared data from WISE and the next generation of multi-object spectroscopy. 


\subsection{High-redshift AGN}

The large sky area of MeerKLASS makes it ideal to search for rare, high- $z$ AGN. These can be used to probe reionization, and can help constrain models of structure formation in the $\sim 1 \mathrm{Gyr}$ period following recombination. LOFAR and MWA hope to detect the $21 \mathrm{~cm}$ transition line at $z \gtrsim 6$, predominantly by searching for signatures via statistical fluctuations in the power spectrum. However, it is also possible to study reionization via $21 \mathrm{~cm}$ absorption, if one can identify sufficiently distant background sources. This is one of the key science questions for the SKA.

Many attempts have been made to find $z>6$ radio sources, mainly using 'steep-spectrum' selection, with the highest redshift radio galaxy at $z=5.19$. The major limitation is the difficulty in obtaining complementary imaging data in the near-IR, which are needed to reliably eliminate low- $z$ sources. The advent of wide-field near-IR surveys (Spitzer, UKIRT, VISTA, WISE) means these data are now available over sufficiently large sky areas to filter out sources at $z<2$, and leave the remaining non-detections for spectroscopic follow-up [e.g. 23].

With the proposed survey area, resolution and depth (required to reliably identify infrared counterparts), we expect to find, in combination with VISTA-VIKING, about ten $z>6$ radio sources with strong emission lines, plus another $\sim 300$ lower-luminosity sources which should still be bright enough for SKA $21 \mathrm{~cm}$ absorption studies.

\subsection{Star-forming galaxies}

The star formation history of the Universe is of great importance to test cosmological theories and also for many applications in astrophysics, such as estimating the build-up of stellar mass in galaxies, constraining the initial mass function, testing models of chemical evolution or constraining the nature of stellar deaths in supernovae and gamma-ray bursts. It is also one of the key science cases to be addressed by the SKA [24]. Radio emission from SF galaxies is produced from free-free emission in HII regions near massive stars and cosmic ray electrons produced by supernova remnants, and offers an unobscured probe of the Universe without the need for extinction corrections.

A survey like MeerKLASS, containing millions of galaxies, will constrain the luminosity functions of AGN and SF galaxies to much higher accuracy, and down to much lower luminosities than previously. Combining the radio data with both spectroscopic and photometric redshifts from optical and near-IR surveys will enable the investigation of how this evolution depends on stellar mass and environment. These are critical for relating the observations to the simulations and thus making important insights into the physics of star formation and the feedback from star formation in lowand high-redshift galaxies.

\subsection{Clustering measurements and AGN environments}

The clustering properties (e.g. bias) of radio sources can be used to provide important constraints on how galaxy formation and evolution are related to the underlying dark matter distribution. MeerKLASS will allow us to extend this work several orders of magnitude deeper, and therefore probe the clustering of faint (sub-mJy) radio sources for the first time.

Clustering measurements can be used to determine the fraction of dark matter haloes of a given mass which host radio-loud AGN (FRIs or FRIIs for example) and also what fraction of these 
exhibit significant star formation activity, providing the information to measure the duty cycle of the AGN activity, which can be used to estimate the timescale of activity and how it relates to the lifetime of star-formation activity in host galaxies. Constraining the duty cycle of AGN, and how this is affected by accretion mode and morphology, is vital to understanding AGN feedback. Furthermore, novel use of cross-correlations between quiescent and active galaxies can be used to investigate how activity depends on halo mass and possibly the age of the dark matter haloes.

The proposed survey will take studies of the clustering of radio sources to the next level: with the large area covered and breadth of ancillary data, we will be able to determine the evolution of the clustering of radio sources as a function of their morphology, luminosity and accretion mode up to $z \sim 2$.

\section{Clusters}

MeerKLASS will observe total intensity and polarization over 4,000 deg ${ }^{2}$ down to a flux limit of $\sim 5 \mu \mathrm{Jy} /$ beam in L-band. Approximately 3,000 galaxy clusters above a $M_{500}$ mass limit of $3 \times 10^{14} M_{\odot}$ are expected within the survey area. This blind survey will produce sensitive radio observations of a statistically significant number of galaxy clusters above this mass limit and over a broad redshift range, $0<z<0.7$.

\subsection{Diffuse cluster emission}

Most diffuse emission studies have been carried out on high-mass $\left(M_{500}>6 \times 10^{14} M_{\odot}\right)$, low redshift $(0.2<z<0.4)$ systems due to the faint nature of the emission and sensitivity limits of current instruments. The MeerKLASS survey is expected to detect a radio halo and/or relic in approximately 200 clusters, lower in mass and over a broad range of redshift, greatly extending the current number of $\sim 65$ sources [25]. This statistically significant sample of diffuse emission is crucial for advancing the understanding of its formation and evolution.

MeerKLASS will measure fluxes and spectral indices across the broad MeerKAT band for each diffuse source. For radio halos, this will allow a separation of the detected population based on their formation theories: re-acceleration during cluster mergers creates turbulent halos, whereas off-state halos are found in less disturbed systems and are generated through hadronic processes. The sensitivity of MeerKAT also allows for an investigation into the evolution and formation of radio halos by studying the rate of occurrence in low mass and/or high redshift systems. Polarization data will be crucial to test the formation models for shock-induced radio relics through magnetic field orientations and inferred Mach numbers. The blind survey will also detect anomalous diffuse sources which do not conform to the current emission characterizations, which will further the understanding of the evolution and properties of diffuse cluster emission. Finally, with the 10 arcsecond L-band resolution of MeerKAT, a statistically significant population of mini-halos should be detected around low-redshift BCGs. This population will be used to begin to investigate whether mini-halos are inherently separate phenomena from radio halos or instead part of the latter's evolutionary process. 


\subsection{Cluster magnetic fields}

The origin of cosmic magnetic fields is still an open field in terms of a full understanding of the processes which generate or amplify them. One of the methods to probe the strength and orientation of magnetic fields in the ICM, necessary for understanding cosmic magnetism, is through Faraday rotation of background sources. With its $5 \mu \mathrm{Jy} /$ beam sensitivity limit, MeerKLASS is expected to detect $\sim 25$ polarized sources per square degree [26], several of which will be in or behind galaxy clusters. Magnetic field studies in clusters over a wide range of mass and redshift will provide crucial statistical information on the properties and evolution of magnetism on cluster scales.

\section{MeerKLASS as an extragalactic HI survey}

MeerKLASS has the potential to become the HI survey with the highest impact among all SKA progenitor surveys. In particular it promises to resolve galaxies over the widest range of galaxy HI masses, allowing us to prepare the full range of SKA science cases studying galaxy formation, dynamics, accretion, and depletion processes. For low- $z$ galaxies, the surface brightness sensitivity will be sufficient to trace also the faint outskirts of galaxies. At the low-mass end it will only be surpassed by MIGHTEE.

With its large survey area and a comparably high sensitivity, MeerKLASS will detect $\mathrm{HI}$ in a few hundred thousand galaxies. Its sensitivity is a factor 3-4 better than the current gold standard ALFALFA, and will hence serve to improve the statistics of integral HI properties of galaxies. A more complete picture of the potential impact of MeerKLASS as a HI survey is evident from Fig. 4, where its detection statistics for resolved galaxies at $z>0.1$ is calculated and compared to various future HI surveys: we assume a very conservative rms noise of $0.5 \mathrm{mJy} / \mathrm{beam}$ over a velocity interval of $20 \mathrm{~km} / \mathrm{s}, 35 \%$ above our assumed continuum sensitivity. To introduce a metric of the survey impact when targeting resolved objects we assume a galaxy to be resolved if there are at least 5 synthesized beams across its major axis extent, the latter defined as the location at which the surface density equals $1 M_{\odot} \mathrm{pc}^{-2}$. We predict the HI extent of each detection using the tight correlation between this quantity and the HI mass, the latter estimated from the HI mass function of [27]. It is clear that MeerKLASS will resolve significantly more galaxies out to larger distances than currently planned HI surveys, providing a unique sample from which to measure the statistics of disk galaxy structure in the local Universe.

According to the metrics presented above, MeerKLASS will be the mother of HI emission line progenitor surveys for the SKA, enabling us to address major research areas: (1) measuring radial gas profiles of galaxies to study galaxy evolution; (2) quantifying the matter distribution in galaxies; (3) studying the intrinsic and mutual spin distribution in galaxies and its dependence on their environment and (4) studying the low column density environment of galaxies to quantify AGN feedback and accretion processes on a statistical basis.

MeerKLASS will produce standard data products to address the above questions: (1) basic emission line parameters; (2) HI emission line maps and data cubes of resolved objects; (3) parametrisations of the extended morphology and kinematics, spin characteristics, rotation curves, and surface density profiles of resolved galaxies and (4) velocity and mass functions. 

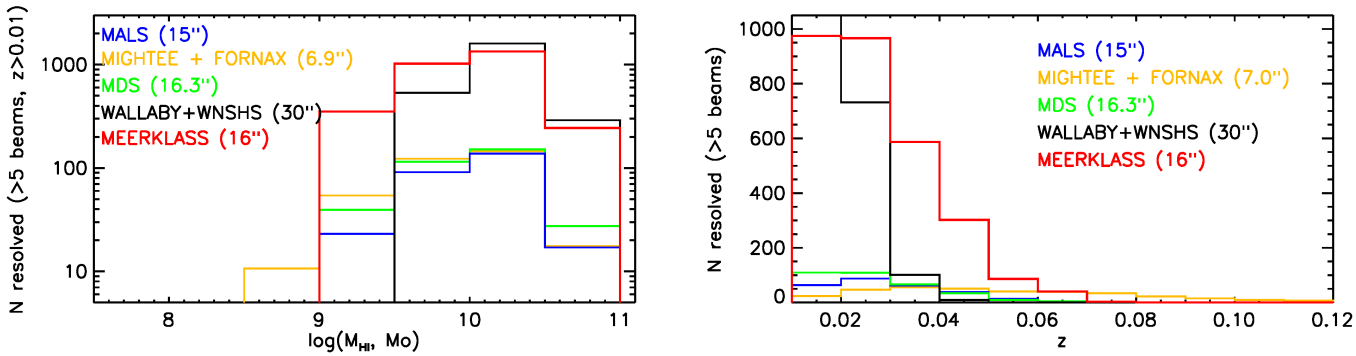

Figure 4: Predicted distributions of resolved galaxy masses and redshifts for various surveys. Left: Separated by HI mass. Right: Separated by redshift. Blue: MALS, yellow: MIGHTEE and FORNAX combined, green: medium-deep APERTIF, black: WALLABY and APERTIF shallow combined, red: MeerKLASS. Numbers in parentheses show angular resolution.

\section{The polarized sky - cosmic magnetic fields}

Current studies of the sub-mJy polarized sky show that it presents questions which cannot be answered by the strong source population alone [28]. The mean fractional polarization of radio sources is anti-correlated with flux density. The faint end of this flux density range contains relatively more objects below the FRI/FRII luminosity boundary than the bright end. This raises a number of questions about the nature and evolution of the faint polarized sources. How is the higher degree of polarization of faint sources related to source structure, radio luminosity, redshift, or environment? Does the trend of increasing polarization continue to lower flux densities? When does it stop? Does the polarization of all AGN increase with flux density, or can we identify a sub-class of AGN that is responsible for this trend?

The fractional polarization and intrinsic polarization angle of a source measure the order and direction of its magnetic field. It is expected that these quantities will be different for distinct classes of object due to the different origin of the emission. In AGN they will mainly be related to the ordered magnetic field in the jets and lobes; for SF galaxies, which are mostly spirals, they are likely to reflect the degree of ordering in the intrinsic disk field. MeerKLASS will have the sensitivity to provide direct detection of the presence and properties of ordered magnetic fields in a vast number of galaxy disks out to intermediate redshifts. At even higher redshifts we will be able to measure statistical polarized source properties to nanoJy levels by stacking the polarized emission at positions corresponding to total intensity detections, and thus trace the emergence of ordered fields in galaxies over cosmic time. With multi-wavelength information we will correlate the radio polarization properties with optical emission line diagnostics, galaxy type and star-formation rates, revealing the relation of the evolution of magnetic fields and the global star formation properties of galaxies.

MeerKLASS at $1.4 \mathrm{GHz}$ will directly detect thousands of polarized sources spanning the transition where SF and normal galaxies become a major component of the polarized population. MeerKLASS will uniquely provide broad-band spectro-polarimetry for sources at $\mu$ y levels over thousands of square degrees. The large bandwidth will provide high precision Rotation Measure (RM) synthesis making it possible to statistically examine the distribution of angular distribution of RMs to search for the imprint of intergalactic fields on galaxies on cosmological scales. We 
will also trace the degree of polarization versus frequency due to internal Faraday rotation of the sources. The amount of depolarization by Faraday dispersion sets apart star-forming galaxies from most AGN powered radio sources, potentially allowing us to separate them using the polarization observations; something which can be cross-checked using the multi-wavelength information.

The bandwidth of the survey will allow Faraday Rotation Measures to be measured with an accuracy better than 2 rad-m ${ }^{-2}$ down to polarized flux densities of $100 \mu \mathrm{Jy}$. From recent JVLA imaging to $1 \mu \mathrm{Jy}$ rms of a $0.1 \mathrm{deg}^{2}$ field at $5 \mathrm{GHz}$, we expect to detect several 10 s of sources per square degree down to $30 \mu \mathrm{Jy}$ at $1.4 \mathrm{GHz}$. The 2-point correlation function of RM will be measured on scales of arc minutes and precision of a few $\mathrm{rad}-\mathrm{m}^{-2}$, precisely the regime where fluctuations from primordial magnetic fields are expected to create detectable signal [29]. This regime of sky density and RM accuracy has not been accessible to date, and will not be surpassed until SKA1.

\section{Summary}

The proposed MeerKAT survey is set to become the leading radio Cosmology survey in preparation towards SKA1-MID. Its design fits well with the MeerKAT set-up both in terms of sensitivity and resolution. The survey will rely both on the dish auto-correlations (single-dish mode) and cross-correlations (interferometer mode). With this, it will provide a 4,000 $\mathrm{deg}^{2}$ map in HI intensity mapping, continuum ( $\sim 5 \mu \mathrm{Jy} \mathrm{rms})$ as well as $\mathrm{HI}$ line and polarization. This will allow us to achieve a wide range of science cases, from cosmology to galaxy evolution, cluster science, HI galaxies and the polarized sky. Many other science cases will certainly take advantage of such a wide survey.

Several challenges will have to be overcome, however, if we want to use this signal in particular for cosmological purposes. For instance, cleaning of the huge foreground contamination, removal of any systematic effects and calibration of the system. Foreground cleaning methods for intensity mapping have already been tested with relative success taking advantage of the foreground smoothness across frequency. The planned fast scanning strategy will allow us to deal with the typical $1 / f$ noise expected in single-dish surveys by taking advantage of the system stability. Using the same scanning strategy for the interferometer calibration will require sophisticated statistical analysis methods in order to take advantage of the usual self-calibration process. Several techniques are currently being developed and we plan to run several tests with the MeerKAT system. Although challenging, this approach has the promise to open a completely new observational window for Cosmology as well as to provide unprecedented high quality data for many other scientific purposes.

Acknowledgements. JF, MJ, RM, EM, KM, MP, PP, MGS, AW and IW are supported by the South African Square Kilometre Array Project and the National Research Foundation. PB is supported by the NASA Postdoctoral Program at the Jet Propulsion Laboratory, California Institute of Technology, administered by Universities Space Research Association under contract with NASA. AP is supported by a Dennis Sciama Fellowship at the University of Portsmouth, and acknowledges the University of Western Cape for hospitality. KK is supported by the Claude Leon Foundation. 


\section{References}

[1] Roy Maartens, Filipe B. Abdalla, Matt Jarvis, and Mario G. Santos. Overview of Cosmology with the SKA. PoS, AASKA14:016, 2015.

[2] M. Santos, P. Bull, D. Alonso, and et al. Cosmology from a SKA HI intensity mapping survey. Advancing Astrophysics with the Square Kilometre Array (AASKA14), art. 19, April 2015.

[3] Matt Jarvis, David Bacon, Chris Blake, Michael Brown, Sam Lindsay, Alvise Raccanelli, Mario Santos, and Dominik J. Schwarz. Cosmology with SKA Radio Continuum Surveys. PoS, AASKA14:018, 2015.

[4] Filipe Batoni Abdalla et al. Cosmology from HI galaxy surveys with the SKA. PoS, AASKA14:017, 2015.

[5] F. B. Abdalla, P. Bull, S. Camera, et al. Cosmology with HI galaxy surveys. In Advancing Astrophysics with the Square Kilometre Array, PoS (AASKA14) 017, December 2014.

[6] Tzu-Ching Chang, Ue-Li Pen, Kevin Bandura, and Jeffrey B. Peterson. Hydrogen 21-cm Intensity Mapping at redshift 0.8. Nature, 466:463-465, 2010. doi: 10.1038/nature09187.

[7] E. R. Switzer, K. W. Masui, K. Bandura, L.-M. Calin, T.-C. Chang, X.-L. Chen, Y.-C. Li, Y.W. Liao, A. Natarajan, U.-L. Pen, J. B. Peterson, J. R. Shaw, and T. C. Voytek. Determination of $\mathrm{z} \sim 0.8$ neutral hydrogen fluctuations using the $21 \mathrm{~cm}$ intensity mapping autocorrelation. MNRAS, 434:L46-L50, July 2013. doi: 10.1093/mnras1/slt074.

[8] K. W. Masui et al. Measurement of $21 \mathrm{~cm}$ Brightness Fluctuations at z 0.8 in Crosscorrelation. Ap. J. L., 763:L20, January 2013. doi: 10.1088/2041-8205/763/1/L20.

[9] Alkistis Pourtsidou, David Bacon, Robert Crittenden, and R. Benton Metcalf. Prospects for clustering and lensing measurements with forthcoming intensity mapping and optical surveys. Mon. Not. Roy. Astron. Soc., 459(1):863-870, 2016. doi: 10.1093/mnras/stw658.

[10] Alkistis Pourtsidou, David Bacon, and Robert Crittenden. HI and cosmological constraints from intensity mapping, optical, and CMB surveys. 2016.

[11] H. Padmanabhan, T. R. Choudhury, and A. Refregier. Theoretical and observational constraints on the HI intensity power spectrum. ArXiv e-prints, July 2014.

[12] Siddharth Satpathy et al. BOSS DR12 combined galaxy sample: The clustering of galaxies in the completed SDSS-III Baryon Oscillation Spectroscopic Survey: On the measurement of growth rate using galaxy correlation functions. Submitted to: Mon. Not. Roy. Astron. Soc., 2016.

[13] T. Abbott et al. The Dark Energy Survey: more than dark energy - an overview. Mon. Not. Roy. Astron. Soc., 2016. doi: 10.1093/mnras/stw641.

[14] P. Bull, P. G. Ferreira, P. Patel, and M. G. Santos. Late-time cosmology with $21 \mathrm{~cm}$ intensity mapping experiments. ArXiv e-prints, May 2014. 
[15] Neal Dalal, Olivier Dore, Dragan Huterer, and Alexander Shirokov. The imprints of primordial non-gaussianities on large-scale structure: scale dependent bias and abundance of virialized objects. Phys. Rev., D77:123514, 2008. doi: 10.1103/PhysRevD.77.123514.

[16] Sabino Matarrese and Licia Verde. The effect of primordial non-Gaussianity on halo bias. Astrophys. J., 677:L77-L80, 2008. doi: 10.1086/587840.

[17] David Alonso, Philip Bull, Pedro G. Ferreira, Roy Maartens, and M. Santos. Ultra largescale cosmology in next-generation experiments with single tracers. Astrophys. J., 814(2): 145, 2015. doi: 10.1088/0004-637X/814/2/145.

[18] José Fonseca, Roy Maartens, and Mário G. Santos. Probing the primordial Universe with MeerKAT and DES. 2016.

[19] S. Camera, M. G. Santos, D. J. Bacon, M. J. Jarvis, K. McAlpine, R. P. Norris, A. Raccanelli, and H. Röttgering. Impact of redshift information on cosmological applications with nextgeneration radio surveys. MNRAS, 427:2079-2088, December 2012. doi: 10.1111/j.13652966.2012.22073.x.

[20] K. McAlpine, M. J. Jarvis, and D. G. Bonfield. Evolution of faint radio sources in the VIDEOXMM3 field. MNRAS, 436:1084-1095, December 2013. doi: 10.1093/mnras/stt1638.

[21] M. Prescott, T. Mauch, M. J. Jarvis, and et al. Galaxy And Mass Assembly (GAMA): the 325 $\mathrm{MHz}$ radio luminosity function of AGN and star-forming galaxies. MNRAS, 457:730-744, March 2016. doi: 10.1093/mnras/stv3020.

[22] E. M. Sadler, R. D. Cannon, T. Mauch, and et al. Radio galaxies in the 2SLAQ Luminous Red Galaxy Survey - I. The evolution of low-power radio galaxies to z 0.7. MNRAS, 381: 211-227, October 2007. doi: 10.1111/j.1365-2966.2007.12231.x.

[23] M. J. Jarvis, H. Teimourian, C. Simpson, D. J. B. Smith, S. Rawlings, and D. Bonfield. The discovery of a typical radio galaxy at $\mathrm{z}=4.88$. MNRAS, 398:L83-L87, September 2009. doi: 10.1111/j.1745-3933.2009.00715.x.

[24] M. Jarvis, N. Seymour, J. Afonso, and et al. The star-formation history of the Universe with the SKA. Advancing Astrophysics with the Square Kilometre Array (AASKA14), art. 68, April 2015.

[25] Z. S. Yuan, J. L. Han, and Z. L. Wen. The Scaling Relations and the Fundamental Plane for Radio Halos and Relics of Galaxy Clusters. ApJ, 813:77, November 2015. doi: 10.1088/0004637X/813/1/77.

[26] L. Rudnick and F. N. Owen. The Distribution of Polarized Radio Sources $>15 \mu \mathrm{Jy}$ in GOODS-N. ApJ, 785:45, April 2014. doi: 10.1088/0004-637X/785/1/45.

[27] A. M. Martin, E. Papastergis, R. Giovanelli, M. P. Haynes, C. M. Springob, and S. Stierwalt. The Arecibo Legacy Fast ALFA Survey. X. The H I Mass Function and $\Omega_{-}$H I from the 40\% ALFALFA Survey. ApJ, 723:1359-1374, November 2010. doi: 10.1088/0004$637 \mathrm{X} / 723 / 2 / 1359$. 
[28] A. R. Taylor. High-Resolution Spectro-Polarimetric Radio Surveys. In D. J. Saikia, D. A. Green, Y. Gupta, and T. Venturi, editors, The Low-Frequency Radio Universe, volume 407 of Astronomical Society of the Pacific Conference Series, page 12, September 2009.

[29] T. Akahori and D. Ryu. Faraday Rotation Measure Due to the Intergalactic Magnetic Field. ApJ, 723:476-481, November 2010. doi: 10.1088/0004-637X/723/1/476. 